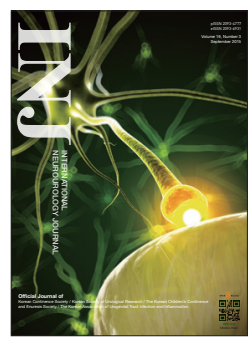

\title{
Meningitis-Retention Syndrome
}

\author{
Dimitrios Basoulis, Maria Mylona, Pantelis Toskas, Dimitris Tsilingiris, Christina Fytili \\ First Propaedeutic Internal Medicine Department, Laiko General Hospital, University of Athens Medical School, Athens, Greece
}

Meningitis-retention syndrome (MRS) is a clinical entity that has recently appeared in the literature. We present the case of a 22-year-old man with fever and headache who, in the course of his hospitalization with a diagnosis of aseptic meningitis, developed acute urinary retention. Fewer than 30 such cases have been described and in several of them, no clear associations with other disorders have been made. In some cases, direct association with viral infection has been proved, and in others, there are indications of an underlying demyelinating condition. To further complicate the issue, various conditions such as Elsberg syndrome and acute disseminated encephalomyelitis, which not only have some similarities but also have some distinct differences, have been placed under the umbrella definition of MRS. In our review, we attempt to address these conditions and better define MRS by establishing diagnostic criteria based on what has thus far been described in the literature.

Keywords: Meningitis; Urinary Retention; Autoimmunity

- Conflict of Interest: No potential conflict of interest relevant to this article is reported.

Meningitis-retention syndrome (MRS) is a newly described clinical entity with features of aseptic meningitis, typically without any clear causative agent, and acute urinary retention. After reviewing the literature, we have discussed the characteristics of this disease.

\section{CASE REPORT}

A 22-year-old male patient presented to the Emergency Department with a three-day history of high-grade fever with rigors and severe headache that did not respond to paracetamol (acetaminophen). Aversion to light or sound was not present. His medical history was unremarkable and he denied taking any medications. On physical examination, the patient was fully conscious (Glasgow Coma Scale 15/15) and his blood pressure was normal (110/60 mmHg), but tachycardia (100 beats/min) and a core temperature of $40.1^{\circ} \mathrm{C}$ were noted. Auscultation of the lungs and the heart did not reveal abnormalities, the abdomen was soft and nontender, and Giordano, Murphy, and McBurney signs were absent. No rash was observed. Brudzinski and Kernig signs were absent, but neck stiffness was present. Fundoscopy did not reveal any papillary edema.

Immediate empirical treatment with ceftriaxone and vancomycin was initiated and a lumbar puncture was performed. Cerebrospinal fluid (CSF) analysis revealed white blood cell (WBC) count of 640 per $\mathrm{mm}^{3}$ with lymphocytic predominance, mild hypoglycorrhachia, and elevated protein levels (Table 1), findings consistent with meningitis. Gram stain, Cryptococcus India ink stain, and cultures of the CSF tested negative. Further CSF testing including polymerase chain reaction for Neisseria meningitidis, Streptococcus pneumoniae, Haemophilus influenzae, Listeria monocytogenes, Pseudomonas aeruginosa, Staphylococcus aureus, herpes simplex virus types 1 and 2, varicella zoster virus, enteroviruses, and tuberculosis was negative. Antibodies against
Corresponding author: Dimitrios Basoulis (iD http://orcid.org/0000-0002-0686-872X First Propaedeutic Internal Medicine Department, Laiko General Hospital, University of Athens Medical School, 17 Agiou Thoma, Athens, Greece E-mail: dimitris.bassoulis@gmail.com / Tel: +30-210-7456343 / Fax: +30-210-7791839

Submitted: May 24, 2015 / Accepted after revision: August 7, 2015
This is an Open Access article distributed under the terms of the Creative Commons Attribution Non-Commercial License (http://creativepenses/by-nc/3.0/) which permits unrestricted non-commercial use, distribution, and reproduction in any medium, provided the original work is properly cited. 
Table 1. A summary of the patient's lumbar puncture results

\begin{tabular}{lrrrc}
\hline \multirow{2}{*}{ Variable } & \multicolumn{4}{c}{ Lumbar puncture } \\
\cline { 2 - 5 } & HD 1 & HD 3 & HD 8 & HD 24 \\
\hline White blood cell $\left(/ \mathrm{mm}^{3}\right)$ & 640 & 625 & 465 & 137 \\
CSF glucose $(\mathrm{mg} / \mathrm{dL})$ & 45 & 48 & 37 & 47 \\
Serum glucose $(\mathrm{mg} / \mathrm{dL})$ & 111 & 110 & 113 & 146 \\
Protein $(\mathrm{g} / \mathrm{L})$ & 1.8 & 1.4 & 1.3 & 0.8 \\
\hline
\end{tabular}

Normal values: $\mathrm{WBCs}<5 / \mathrm{mm}^{3}$; protein, $0.2-0.4 \mathrm{~g} / \mathrm{L}$; glucose $>2 / 3$ of serum.

HD, hospital day; WBC, white blood cell; CSF, cerebrospinal fluid.

West Nile virus were not detected in the CSF or serum. All blood cultures were negative. Apart from mild leukocytosis with neutrophil predominance, complete blood count test and analysis of biochemical parameters did not reveal any abnormalities. C-reactive protein and erythrocyte sedimentation rate were significantly elevated. The patient remained febrile with nuchal rigidity and the fever only partially responded to steroid pulses, such as bolus administration of 250-mg hydrocortisone. A computed tomography (CT) scan of the brain revealed no pathological findings.

On the third day of hospitalization, in the absence of a diagnosis, a new lumbar puncture was performed with similar findings and a trend towards slight improvement (Table 1). Serology for other infectious microorganisms such as human immunodeficiency virus, Toxoplasma gondii, Brucella melitensis, Borrelia burgdorferi, and Mycoplasma pneumoniae also proved negative. The possibility of autoimmune disease involving the central nervous system was considered remote owing to the absence of other systemic manifestations and negative tests for autoantibodies (antinuclear antibodies, antineutrophil cytoplasmic antibodies). Antibiotic treatment was discontinued.

On the sixth day of hospitalization, with the patient still febrile and without any neurological changes, he reported hesitancy and straining to urinate in spite of normal bladder sensation. He exhibited mild suprapubic discomfort on palpation. Microscopic examination of the urine did not reveal any abnormalities and the urine culture was sterile. Tamsulosin $(0.4 \mathrm{mg}$ daily) and distigmine (5 $\mathrm{mg}$ twice daily) did not relieve the aforementioned symptoms. Magnetic resonance imaging (MRI) of the brain and the spinal cord was performed, revealing no pathological findings.

Despite the persistence of fever, a new lumbar puncture on the eighth day of hospitalization revealed a decrease in WBCs and CSF protein levels, implying a trend towards spontaneous recovery (Table 1). Four days later, however, the patient complained of abdominal pain and complete inability to urinate. Urinary retention was diagnosed and a 16-Fr Foley catheter was inserted, retained $850 \mathrm{~mL}$ of clear urine. On urodynamic study, the patient first reported sensation at $120 \mathrm{~mL}$ and exhibited a bladder capacity of $330 \mathrm{~mL}$, both values within normal range. When asked to void, he demonstrated a detrusor pressure $\left(\mathrm{P}_{\mathrm{det}}\right)$ of $28 \mathrm{~cm} \mathrm{H}_{2} \mathrm{O}$ at a peak flow (Qmax) of only $10 \mathrm{~mL} / \mathrm{sec}$, values indicative of detrusor underactivity [1]. The patient's prostate was found to be normal upon ultrasound examination.

On the 24th day of hospitalization, fever was still present, though better tolerated by the patient. Further CSF testing on that day was consistent with the patient's clinical course (Table 1). The patient gradually became afebrile and was discharged several days later. After one week, the Foley catheter was removed and the patient was able to urinate without assistance. On re-evaluation after one month, the patient remained afebrile with no residual neurological deficit.

\section{DISCUSSION}

We present an interesting case of aseptic meningitis further complicated by urinary retention. Few such cases have been published during the past few years. This combination of symptoms has thus far been termed MRS and no clear associations with other disorders have been made.

The patient presented with symptoms resembling those described in the published literature. Most patients are young, healthy adults and present with neurological symptoms including headache, dizziness, drowsiness, and fever, and with signs indicative of meningitis, including nuchal rigidity and positive Kernig and/or Brudzinski signs. Urinary retention was observed a week after the onset of neurological symptoms. In majority of the published cases, CSF analysis revealed lymphocytic pleocytosis, elevated protein levels, and mildly decreased glucose levels [2]. In most cases, no cause is determined [2].

Thus far, no clear associations regarding MRS have been proven. Many theories have attempted to explain the pathophysiology of this syndrome, including sacral myeloradiculitis due to direct viral inflammation, a postinfectious inflammatory demyelinating syndrome (acute disseminated encephalomyelitis, ADEM), and spinal shock secondary to meningeal irritation $[3,4]$.

Urodynamic studies in these patients showed an acontractile type of neurogenic bladder with retention and impaired sensation during the filling phase. It is suggestive that the underactive 
detrusor muscle is not able to achieve intravesical pressures high enough to initiate micturition [2,5]. Neurogenic bladder associated with neurological disease is typically caused by lower motor neuron lesions that result in an underactive detrusor. Elsberg syndrome (infectious sacral polyradiculitis) is a neurological disease characterized by lower motor neuron damage. Syndrome such as MRS is characterized by urinary retention and CSF pleocytosis [2,6]. However, there are some key differences between Elsberg syndrome and MRS. Symptoms of Elsberg syndrome are a result of a presumed herpetic viral inflammation leading to myeloradiculitis without meningitis. Thus, neurological signs and symptoms of meningitis are absent, and the results of the CSF analysis are not as significant as with MRS. Fever is also absent [6]. Symptoms of Elsberg syndrome also include herpetic genital vesicles, hypoesthesia, muscle weakness, and lower back pain due to radiculitis. In Elsberg syndrome patients, activation of the herpes virus in the dorsal root ganglia with axonal spread to the spinal cord can be seen as hyperintense T2 lesions on an MRI scan of the spinal cord. In MRS patients, MRI of the spinal cord reveals no lesions, although there has been some speculation regarding reversible lesions in the splenium of the corpus callosum [7].

Infrequently, an underactive detrusor muscle can be caused by upper motor neuron lesions in the brain or the spinal cord. A prime example of this condition is ADEM, in which a demyelinating syndrome develops after a presumed viral infection. In a small number of patients, the lower urinary tract is the only affected area [8]. It is possible that in some ADEM patients, the innervation of the lower urinary tract is selectively vulnerable [9]. In some cases, myelin basic protein and oligoclonal bands can be detected in the CSF of MRS patients, suggesting that MRS may be a mild, localized form of ADEM [9]. However, ADEM patients present with signs of encephalitis and myelitis. They also commonly show abnormalities (white matter demyelinating lesions) during MRI of the brain and less frequently, of the spinal cord [10].

It is necessary to define MRS in a more precise manner in order to differentiate it from other similar conditions. In most cases reported as MRS, there are no encephalitic signs, distinguishing it from ADEM. Moreover, excluding occasional splenial lesions, MRI of the brain revealed no abnormalities, thereby distinguishing this condition from demyelinating diseases. Lack of leg numbness and paresthesias also helps to differentiate MRS from Guillain-Barre syndrome, polyneuropathies, and conditions affecting the lower motor neurons [2].

We believe that MRS is indeed a mild form of ADEM trig- gered by a viral infection, which in some cases cannot be identified. In the case of our patient, given the low CSF glucose levels and the duration of his meningitis signs and symptoms, we believe that the cause was lymphocytic choriomeningitis virus. However, we were unable to verify our hypothesis.

In this article, we have described the history and clinical findings of MRS, a syndrome combining aseptic meningitis and acute urinary retention. It is important to differentiate MRS from other disorders, in that MRS is a benign and self-limiting condition despite the time required for its complete resolution [2]. Physicians must explain to MRS patients that no patient thus far has developed permanent neurological damage and all have been able to resume their normal activities.

\section{REFERENCES}

1. Osman NI, Chapple CR, Abrams P, Dmochowski R, Haab F, Nitti V, et al. Detrusor underactivity and the underactive bladder: a new clinical entity? A review of current terminology, definitions, epidemiology, aetiology, and diagnosis. Eur Urol 2014;65:389-98.

2. Sakakibara R, Kishi M, Tsuyusaki Y, Tateno A, Tateno F, Uchiyama T, et al. Meningitis-retention syndrome: a review. Neurourol Urodyn 2013;32:19-23.

3. Caplan LR, Kleeman FJ, Berg S. Urinary retention probably secondary to herpes genitalis. N Engl J Med 1977;297:920-1.

4. Krishna A, Devulapally P, Ghobrial I. Meningitis retention syndrome. J Community Hosp Intern Med Perspect 2012;2:15761.

5. Kim TW, Whang JC, Lee SH, Choi JI, Park SM, Lee JB. Acute urinary retention due to aseptic meningitis: meningitis-retention syndrome. Int Neurourol J 2010;14:122-4.

6. Eberhardt O, Kuker W, Dichgans J, Weller M. HSV-2 sacral radiculitis (Elsberg syndrome). Neurology 2004;63:758-9.

7. Tascilar N, Aydemir H, Emre U, Unal A, Atasoy HT, Ekem S. Unusual combination of reversible splenial lesion and meningitis-retention syndrome in aseptic meningomyelitis. Clinics (Sao Paulo) 2009;64:932-7.

8. Panicker JN, Nagaraja D, Kovoor JM, Nair KP, Subbakrishna DK. Lower urinary tract dysfunction in acute disseminated encephalomyelitis. Mult Scler 2009;15:1118-22.

9. Tateno F, Sakakibara R, Sugiyama M, Takahashi O, Kishi M, Ogawa $\mathrm{E}$, et al. Meningitis-retention syndrome: first case of urodynamic follow-up. Intern Med 2011;50:1329-32.

10. Sakakibara R, Yamanishi T, Uchiyama T, Hattori T. Acute urinary retention due to benign inflammatory nervous diseases. J Neurol 2006;253:1103-10. 